\title{
Current understanding of the role and regulation of miRNAs in Burkitt lymphoma
}

This article was published in the following Dove Press journal: Blood and Lymphatic Cancer:Targets and Therapy

\author{
Alessandro Davide Videtta \\ Valeria Malagnino ${ }^{2}$ \\ Giulia De Falco 3 \\ 'I83th Paratroop Regiment "Nembo" \\ Italian Army, Pistoia, Italy; ${ }^{2}$ Onco- \\ Haematology Department SS. \\ Annunziata Hospital, Taranto, Italy; \\ ${ }^{3}$ Faculty of Science and Engineering, \\ School of Biological and Chemical \\ Sciences, Queen Mary University of \\ London, London, UK
}

\begin{abstract}
Since its discovery in 1958, Burkitt lymphoma (BL) has been extensively studied and has become a model for tumorigenesis, but its pathogenesis has not been completely explained and understood yet. The aim of this review was to summarize the current knowledge about BL and, in particular, to discuss the role of miRNAs in its pathogenesis and their possible use as diagnostic and prognostic indicators. The impact of viral-encoded miRNAs is also discussed, with the Epstein-Barr infection being almost invariably detected in the endemic variant of this tumor.
\end{abstract}

Keywords: Burkitt lymphoma, miRNAs, $M Y C$

\section{Introduction \\ Discovery of Burkitt lymphoma (BL)}

BL was first described by Denis Burkitt in $1958^{1}$ during a field trip to sub-Saharan Africa and was subsequently named after him. The British surgeon observed that this particular tumor had a very high incidence in this geographic region, referred to as the "lymphoma belt" of Africa, in which other infectious diseases are also very common, such as malaria and arboviral infections. ${ }^{2,3}$ Nevertheless, even though $\sim 60$ years have passed since the first description, it is not clear yet what impact, if any, these infectious diseases may have in driving BL in endemic areas. ${ }^{4}$

$\mathrm{BL}$ has been referred to as the "Rosetta stone" of cancer because it is the first tumor for which a viral association has been described (with the Epstein-Barr virus $[\mathrm{EBV}]),{ }^{5}$ the first tumor in which a specific chromosomal translocation has been identified (involving the $M Y C$ proto-oncogene) ${ }^{6,7}$ and the first tumor successfully treated by chemotherapy. ${ }^{8}$ For this reason, BL has a very important "historic" role, as it has improved our understanding of the molecular mechanisms happening in cancer, and it is still considered a model for tumorigenesis.

The EBV was isolated by Sir Anthony Epstein in 1964 from a BL-derived cell line, ${ }^{5}$ and now clear evidence highlight that this virus is not simply a bystander, but it actively promotes transformation through its encoded genome products. ${ }^{9}$

\section{Classification of BL} School of Biological and Chemical Sciences, Queen Mary University of London, Room 3.I2 Fogg Building, Mile End Campus, London EI 4NS, UK Tel +442078823206

Email giulia.defalco@qmul.ac.uk
According to the World Health Organization classification, BL can be defined as a "single morphological and clinical entity, with variations in clinical presentation" ${ }^{10}$ Three subtypes of this tumor have been described, namely, the endemic (eBL), the sporadic (sBL) and the immunodeficiency-associated form (ID-BL or human immunodeficiency 
virus [HIV]-BL) that differ in geographic distribution and the extent of association with viruses, with EBV being the most relevant. ${ }^{10}$ Despite this distinction, these variants can still be considered the same entity as they all share the same clinical presentation and the same molecular profile, ${ }^{11,12}$ with some differences in their miRNA profile. ${ }^{13}$ The hallmark of $\mathrm{BL}$ is the constitutive activation of the $M Y C$ proto-oncogene, which leads to deregulated and increased expression of the Myc protein. In the vast majority of cases, this imbalance is achieved through a chromosomal translocation, which puts the $M Y C$ gene, mapping on chromosome 8, under the transcriptional control of immunoglobulin (Ig) gene promoters. When these promoters are very active, following a translocation, there is a strong and sustained expression of the $M Y C$ gene, with a resulting upregulation of its protein product. ${ }^{6,7}$ Three different translocations have been described involving different Ig loci $(\mathrm{t}[8 ; 14]$, $\mathrm{t}[8 ; 2]$ and $\mathrm{t}[8 ; 22])$, of which the $\mathrm{t}(8 ; 14)$ is the most frequently observed in $\mathrm{BL}(\sim 80 \%$ of $\mathrm{BL}$ cases). ${ }^{6,7}$ However, in the past 2 decades, it has been observed that BL cases overexpressing Myc, but lacking an identifiable translocation do exist. ${ }^{14}$ These cases are indistinguishable from translocated BLs with respect to clinical presentation and share the same gene expression profile, ${ }^{11}$ though they present with differences in their miRNA signature, as explained later in this review. ${ }^{15}$ This suggests that alternative pathogenetic mechanisms responsible for increased levels of the Myc protein exist, besides the chromosomal translocation. Interestingly, no differences regarding the presence of $M Y C$ translocation are observed among the different clinical variants of BL. BL immunophenotype shows the expression of B-cell-associated antigens (eg, CD19, CD20 and CD22) and additional proteins such as CD10, BCL6, CD38, CD77 and CD43, which suggests its derivation from late germinal center (GC) cells. ${ }^{10}$ However, it has been postulated that sBL and eBL derive from GC cells at different stages of differentiation, as they show a different pattern of Ig hypermutation and signs of antigen selection. ${ }^{16}$ Based on this observation, it is possible that sBL derives from early GC cells (centroblasts), whereas eBL derives from late GC cells (centrocytes), as the latter show a higher number of somatic hypermutations in the Ig genes. ${ }^{16}$

\section{Clinical variants of BL: endemic, sporadic and immunodeficiency associated}

Despite BL showing a very homogeneous molecular profile, ${ }^{11,12}$ differences can be pinpointed regarding the geographic distribution of this tumor and association with EBV. The endemic form has a very high incidence in Equatorial
Africa, where other climatic conditions and infectious agents may possibly act as cofactors, though it is still debated at which extent and through which mechanisms. ${ }^{4}$ The endemic form of $\mathrm{BL}$ is preferentially observed in young children (especially males), with a peak incidence of 4-7 years. It most commonly presents in extranodal sites, with the jaw and other facial bones being very frequently affected. Most importantly, the degree of association of this clinical variant with EBV is extremely high, the virus being detected in $\sim 100 \%$ of eBL cases. ${ }^{10}$ Such a strong association suggests an active involvement of EBV in Burkitt pathogenesis, and recent literature proves an important role for its encoded products, ${ }^{9}$ although there is still a lot to uncover. Based on the simple association with EBV, 90-95\% of the world population test positive for $\mathrm{EBV}$, but BL incidence is much lower. This suggests that other factors may be required for BL pathogenesis.

The sporadic form occurs anywhere in the world and is histologically identical to eBL. It is still a pediatric disease, accounting for $\sim 30 \%$ of childhood lymphomas (and $1 \%-2 \%$ of all lymphomas), and yet has a higher incidence in males, though with a higher median age of incidence (12 years). Another difference is that, despite sBL also showing an extranodal presentation, jaw tumors are less frequent, with the gastrointestinal tract being the most common site of involvement. Very interestingly, EBV is less commonly associated with sBL, being detected only in $\sim 20 \%-30 \%$ of cases. ${ }^{10}$

As far as the immunodeficiency-related form is concerned, this clinical variant is particularly frequent in HIV-positive individuals, accounting for one-third of HIVassociated lymphomas, and it is therefore also referred to as HIV-related BL. This tumor is mostly observed in adults; it mainly shows a nodal presentation, with a generalized lymph node involvement and the bone marrow and central nervous system involvement also being common. Extranodal disease is possible, but it is much less frequent than in endemic and sporadic forms. The immunodeficiency-related variant shows a variable extent of association with EBV, ranging from $30 \%$ to $90 \%$ of all cases. ${ }^{10}$ Noteworthy, this form is not only observed in HIV-infected patients but also in other types of immunodeficiency (ie, posttransplant), although BL may be the first disease to manifest in HIV-infected individuals, as it occurs in patients with a still high $\mathrm{CD}^{+}$ count and not having overt acquired immunodeficiency syndrome yet. This evidence suggests that, despite its role as an oncogenic virus still being debated, HIV may actively contribute to $\mathrm{BL}$ pathogenesis either indirectly, through a 
continuous antigenic stimulation, or directly, through its encoded genome products. ${ }^{17}$

\section{MYC deregulation in $\mathrm{BL}$}

The $M Y C$ proto-oncogene is a powerful transcription factor and plays very important physiological functions, being involved in the control of proliferation, cell growth, metabolism, apoptosis and differentiation. ${ }^{18}$ It belongs to the Myc family of transcription factors (comprising $M Y C, M Y C N$ and $M Y C L$ ), of which $M Y C$ is the best characterized. It was originally identified because of its homology with $v-M Y C$, the transforming gene of the MC29 avian leukemia virus, ${ }^{19}$ and subsequently, its deregulation has been reported in a wide range of human tumors, though its activation may be achieved through different pathogenetic mechanisms. ${ }^{20}$ $M Y C$ may either induce or repress transcriptional activation, thus regulating the expression of many downstream targets and consequent biological pathways. ${ }^{21}$ To carry out transcriptional control, the Myc protein binds to other transcriptional regulators such as Max or Mnt, Mxd1-4 (Mad1, Mxi1, Mad3 and Mad4) and Mga, which influence Myc transcriptional regulation pushing toward either activation or repression of downstream targets. Proliferative stimuli induce the expression of $M Y C$ and lead to the formation of Myc:Max heterodimers and concomitant activation of target gene expression, thus resulting in transcriptional activation. ${ }^{21}$ Other transcription factors may compete with Max for binding to Myc (Mnt, Mxd1-4 and Mga), thus resulting in transcriptional repression. ${ }^{22}$ In addition, Myc can bind to transcription factors Sp1 and Miz1 and may interfere with their transcriptional activator capability. The complex Myc-Miz1 recruits DNA methyl transferase 3a and histone deacetylase 3 to gene promoters, leading to DNA cytosine methylation and histone deacetylation, therefore causing gene expression silencing. The Myc-Miz1 complex can, therefore, induce the formation of heterochromatin on its target sites and function as a transcriptional repressor complex. ${ }^{22}$ Due to its key involvement in transcription regulation, Myc expression and function must be tightly controlled.

Pathological activation of $M Y C$ associated with gain-offunction mutations has been commonly described in cancer. It can be due to chromosomal translocations leading to promoter rearrangements (as observed in most BL cases), gene amplifications (commonly reported in breast cancer), virusmediated insertional mutagenesis (less commonly observed, due to random insertions of viruses within the genome) and Myc protein stabilization, mainly due to genetic mutations. ${ }^{21}$ Though all these mechanisms are possible in BL, the most commonly observed cause of Myc deregulation is usually the presence of a balanced translocation involving chromosome 8, where $M Y C$ maps, and different partners. Mutations of the $M Y C$ coding sequence have also been described in $\mathrm{BL}$, as well as variations in its copy number, but they occur in a minority of $\operatorname{cases}^{23}$ and do not seem to account for the main reason of $M Y C$ upregulation. Nevertheless, in the last few years, a few cases of BL in which none of the abovementioned mechanisms could possibly explain Myc hyperexpression have been described, and alternative pathogenetic mechanisms were investigated. No matter what is leading to $M Y C$ deregulation, the consequence is an increased expression of its protein product which is invariably associated with genomic instability, uncontrolled cell proliferation, escape from immune surveillance and malignant transformation. ${ }^{20}$

\section{Additional genetic lesions in $\mathrm{BL}$}

Despite $M Y C$ deregulation being absolutely crucial for $\mathrm{BL}$ pathogenesis, $M Y C$ imbalance is not the only genetic lesion identified in BL and other recurrent or sporadic lesions have also been described. Several genes have been reported to be mutated in BL, such as the tumor suppressors $I D 3^{24,25}$ or $T C F 3,{ }^{26}$ whose mutations seem to be quite common in $\mathrm{BL}$, especially in its sporadic variant. Additional mutations have been detected in genes belonging to the PI3K pathway, ${ }^{27}$ in the SWI/SNF family members and in ARIDIA and SMARCA4A among others, which suggest functional alterations of the nucleosome remodeling complex. ${ }^{28}$ In addition, genes whose mutations have already been described in other B-cell lymphomas, such as $M Y C$ itself, $D D X 3 X, C C N D 3$ and $F B X O 11$, among others, have also been reported to be mutated in BL. ${ }^{25,26}$ Recently, mutations of particular genes have been reported to occur at a different frequency in eBL and sBL, and a correlation between a distinct mutation pattern and the existence of some viral infections has been suggested. ${ }^{29}$ This observation may indicate that different pathogenetic mechanisms may exist in eBL and $\mathrm{sBL}$ and again suggests that viruses may play an active role in contributing to BL development.

Genetic lesions other than point mutations have also been described in BL. In particular, copy number alterations including gains of 1q, 9q, 12q, 13q, 20q, 22q and Xq and losses of $4 \mathrm{q}, 13 \mathrm{q}$ and $17 \mathrm{p}$ have been reported..$^{25,30-34}$ Additionally, trisomy $1 \mathrm{q}^{31}$ and tetrasomy $1 \mathrm{q}$ have also been described. ${ }^{32}$ Interestingly, gains of $11 \mathrm{q}$ have been frequently reported in a subset of tumors resembling BL but lacking MYC translocation, ${ }^{35}$ although it is debated whether such cases should be diagnosed as BLs or rather as different aggressive B-cell tumors with a Burkitt-like presentation. ${ }^{35}$ 
In addition, uniparental disomy, whose role has been recently highlighted in cancer, ${ }^{36}$ does not seem to play a major role in the pathogenesis of BL. ${ }^{37}$

\section{Myc upregulation due to impairment of post-transcriptional regulation: the role of miRNAs}

Regulation of gene expression must be finely tuned and may be controlled at different levels. Transcriptional regulation can be achieved by epigenetic changes, which regulate the accessibility of specific DNA sequences through methylation of histones and DNA, thus determining a conformational change in the chromatin and preventing the expression of genes when their function is not required. Regulation of gene expression is further controlled at the post-transcriptional level, when a certain mRNA has already been transcribed, but translation into the correspondent protein product is impaired. Post-transcriptional regulation is achieved by small non-coding RNAs, of which miRNAs have been intensively studied in the last few years.

miRNAs were isolated for the first time from Caenorhabditis elegans in $1998,{ }^{38}$ and since the first observation, they have been described in a wide range of organisms, including humans. They are small sequences of non-coding RNA that, in their mature form, have sizes of 18-24 bp, though they are processed from longer precursors during a maturation process that also shuttles them from the nucleus to the cytoplasm..$^{38}$ After maturation, the miRNAs bind to complementary mRNA sequences and prevent their translation into the corresponding proteins. Depending on their degree of complementarity with the target mRNAs, they can either lead to mRNA degradation, when there is a perfect pairing, or simply to translation impairment, if there are mismatches in the pairing. However, no matter whether the mRNA is degraded or not, the consequence of miRNA-mRNA binding is that production of the protein coded by that particular mRNA is prevented. This further level of regulation allows shutting down the expression of specific genes even when their transcription into an mRNA has already taken place. More importantly, it is worth mentioning that a single miRNA can target hundreds of mRNAs based on a short sequence complementarity. Deregulation of a single miRNA may, therefore, result in deregulated expression of many genes, thus affecting several distinct pathways and biological functions within the cell. With the function of miRNA being so delicate in tuning the gene expression, their function must be strictly controlled as its imbalance might lead to disturbance of gene expression. Deregulation of miRNA expression and function has, therefore, been reported in a plethora of human diseases, including cancer. ${ }^{39}$

\section{MYC and miRNAs control each other's expression: the existence of a feedback regulatory loop}

We have already mentioned the importance of $M Y C$ as a transcription regulator. With its capability to bind to target sequences on DNA, Myc can control the expression of coding as well as non-coding regions in the DNA, including genes and miRNAs. Myc is known to regulate the expression of $\sim 60$ miRNAs, ${ }^{40,41}$ either positively or negatively influencing their expression (Figure 1). As a transcriptional activator, Myc can induce the expression of selected miRNAs, of which the miR-17-92 cluster is the prototypical example, ${ }^{42}$ thus influencing the expression of miRNA downstream target genes and eventually influencing the related biological processes (Figure 2A). Induction of miR-17-92 by MYC has been previously reported not only in BL and other B-cell tumors ${ }^{42}$ but also in various different tumors including breast, lung, colon, stomach and prostate (for a review, see Bui and Mendell ${ }^{43}$ ). Inhibition of downstream target genes of the miR-17-92 cluster enhances tumorigenicity by boosting cell proliferation, tumor cell survival and angiogenesis, along with metabolic reprogramming. ${ }^{43}$ Very interestingly, among the downstream targets of this cluster, there are tumor suppressors such as PTEN and BIM, the first gene being an antagonist of PI3K activity and the latter having a proapoptotic function. ${ }^{43-46}$ Overexpression of miR-17-92 induced by Myc, therefore, results in loss of regulatory control on cell growth mediated by these tumor suppressors. A recent study investigated the expression of each member of this cluster in BL and analyzed whether there was a correlation between their expression and prognosis of BL. ${ }^{47}$ The results of this study indicated that miR-17 and miR-20a were highly expressed in $\mathrm{BL}$ and determined lack of expression of the Bim protein. ${ }^{47}$ In addition, a significant correlation between high levels of miR-17 and poor overall survival was also recorded, thus indicating the influence of miRNA expression as a prognostic value in $\mathrm{BL} .{ }^{47}$

At the same time, Myc can also repress the expression of specific miRNAs (ie, the miR-29 family), thus leading to increased expression of miRNA target genes and imbalance of cellular pathways (Figure 2A). However, MYC itself is a gene and, therefore, its expression is also controlled at the post-transcriptional level by miRNAs, of which probably the best studied is the let-7 family. ${ }^{48} M Y C$ expression, both at the transcriptional and post-transcriptional levels, is therefore 


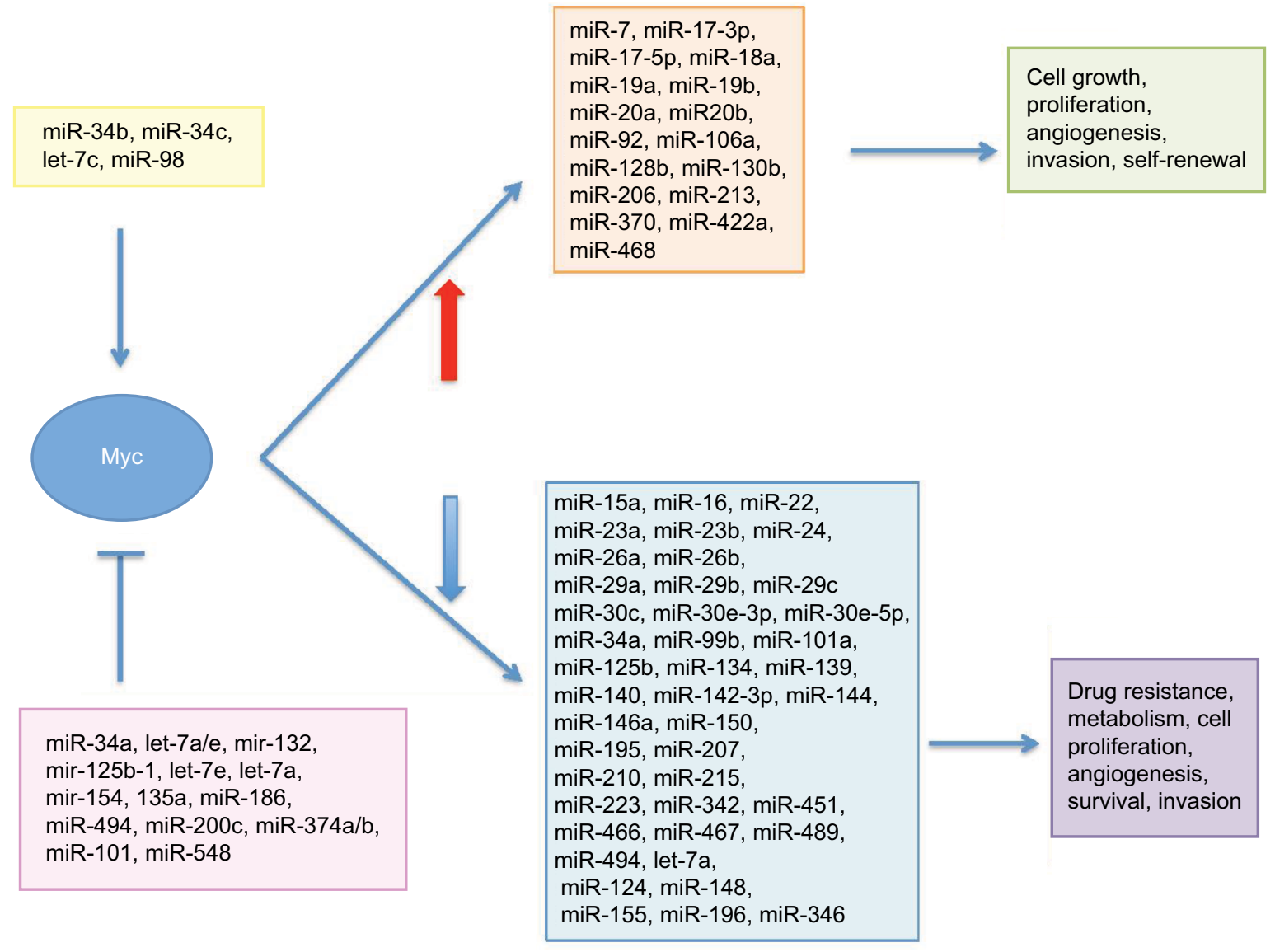

Figure I An overview of MYC-miRNA regulatory loop and related pathways.

Note: miRNAs upregulated by Myc are indicated by the red arrow and the downregulated miRNAs are indicated by the blue arrow.

controlled through different mechanisms to ensure that this protein will be produced only when its function in the cell is needed. Thus, miRNAs-MYC form a feedback regulatory loop controlling each other's expression in an inverse and reciprocal manner (Figure 1). When this miRNAs-MYC autoregulation fails, the expression of $M Y C$ and miRNAs is no longer regulated and may result in diseases and cancer.

A recent study reported the upregulation of the $Y Y 1$ gene, which is an oncogenic transcription factor able to induce $M Y C$ expression, in $\mathrm{BL}$ as a consequence of downregulation of specific miRNAs. ${ }^{49}$ This transcription factor was previously found to be upregulated in other non-Hodgkin's lymphomas (NHLs) $^{50,51}$ and plays a role in resistance to chemotherapy and immunotherapy in NHL cell lines. ${ }^{52}$ YY1 can also act as a transcriptional repressor of tumor suppressors such as p16, p27, p73 and p53. ${ }^{53-55}$ In particular, its inhibitory effect on p53 is related to evasion from apoptosis ${ }^{56}$ which may be crucial for transformed cells, pointing at $Y Y 1$ as an indicator of aggressiveness in NHLs. Very interestingly, upregulation of $Y Y 1$ reported in this study is a consequence of repression of specific miRNAs, some of which, such as has-miR-363 and
hsa-miR-200a, are among the top 20 miRNAs repressed in BL, thus reinforcing its functional role in the pathogenesis of BL. ${ }^{49}$

\section{Exploring the MYC-miRNA interaction in BL: sustaining Myc hyperexpression in the absence of a translocation}

Approximately $10 \%$ of BL cases lack an identifiable $M Y C$ translocation, but do express the Myc protein at a level comparable to $M Y C$-translocated BL cases. ${ }^{57}$ This observation prompted many scientists worldwide to explore alternative pathogenetic mechanisms that could explain a higher expression of Myc in the absence of genetic lesions, either translocation or copy number alterations. Given the functional relationship between $M Y C$ and miRNAs, one possible scenario to explore was to investigate whether there was an imbalance in $M Y C$-regulating miRNA expression that could eventually explain increased Myc protein levels. A pioneer study published in 2008 compared the expression of six miRNAs predicted to target $M Y C$ (hsa-miR-155, has-miR-30a-3p, hsamiR-34b, hsa-let-7c, hsa-let-7a and hsa-miR-98) between BL cases carrying or not an $M Y C$ translocation. ${ }^{57}$ The results of 
A

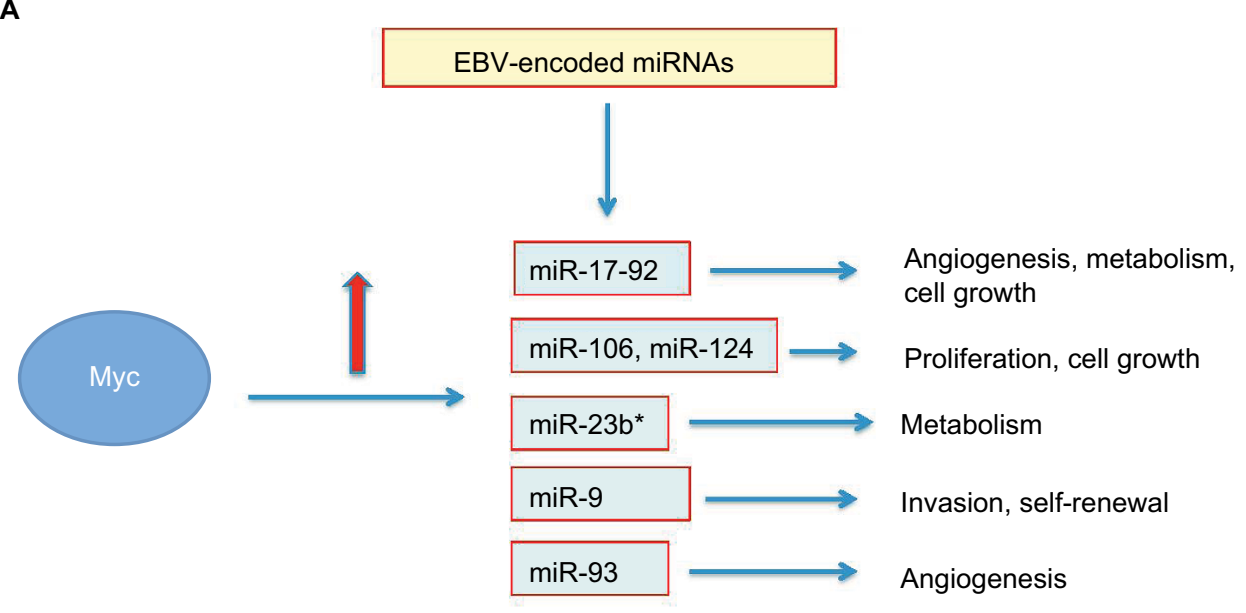

B

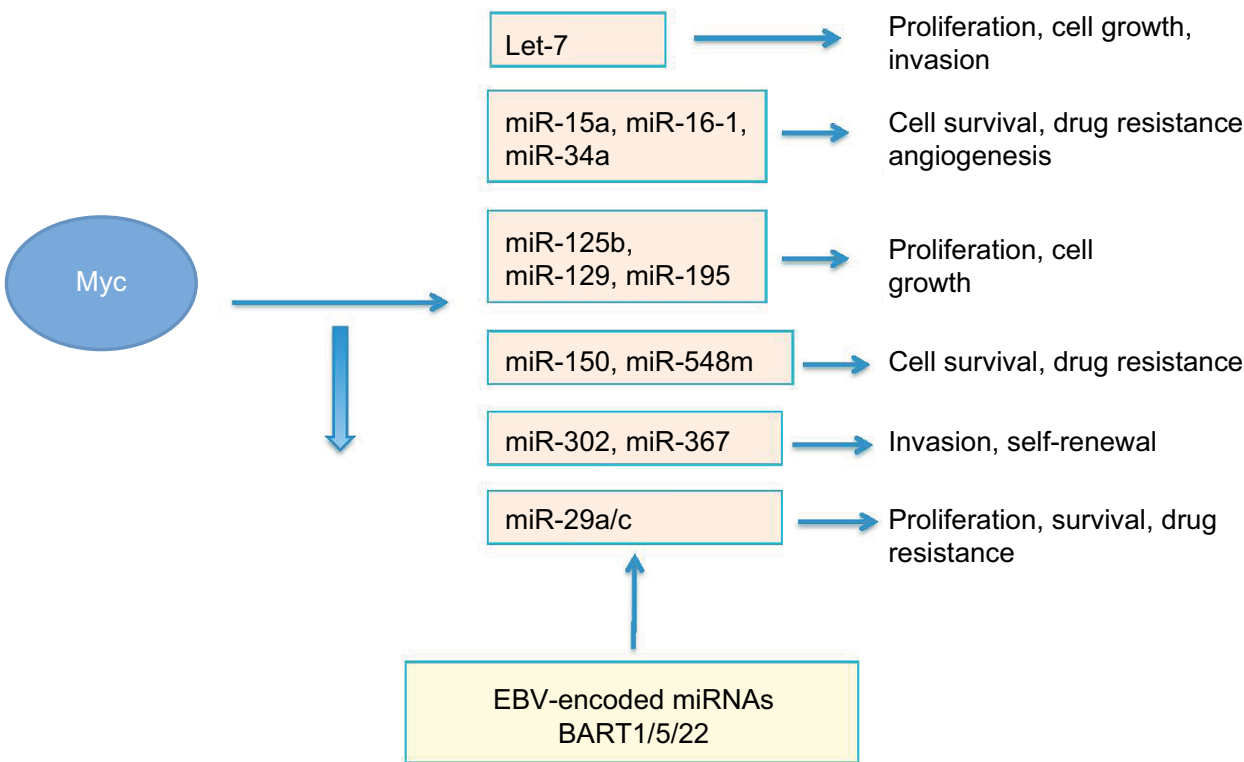

Figure 2 An overview of the pathways affected by Myc-regulated miRNAs.

Notes: (A) Myc-induced miRNAs and their regulated pathways. EBV-encoded miRNAs may compete with the miR-17-92 cluster for regulation of the same target genes. (B) Myc-repressed miRNAs and their regulated pathways. EBV-encoded BARTs may compete with cellular miR-29 family members.

Abbreviation: EBV, Epstein-Barr virus.

this study highlighted a diminished expression of two of them (hsa-let-7a and hsa-miR-34b), thus suggesting that increased expression of Myc may be a consequence of downregulation of specific miRNAs. ${ }^{57}$ However, of even greater interest was the observation that hsa-miR-34b was downregulated only in BL cases lacking the translocation, whereas reduced expression of hsa-let-7a was observed in all BL cases, irrespective of the translocation status. This observation suggested the alteration of hsa-miR-34b as potentially responsible for Myc hyperexpression in the absence of any genetic lesions. ${ }^{57} \mathrm{~A}$ later study of the same group identified hsa-miR-9* as a second miRNA specifically downregulated only in BL cases lacking MYC translocation. ${ }^{58}$ This observation was of particular interest because hsa-miR-9* does not directly target $M Y C$, but may indirectly regulate its expression through E2F1, whose expression is induced by Myc, ${ }^{59,60}$ and that in turn activates $M Y C$ expression through a feedback autoregulatory loop that also involves the miR-17-92 cluster. ${ }^{61-63}$ Hsa-miR-9* downregulation observed in BL cases lacking $M Y C$ translocation could determine the upregulation of E2F1, which then increases and sustains $M Y C$ expression. The complete miRNA expression profile was then investigated in BL cases with or without $M Y C$ translocation and differential expression of four miRNAs (hsa-miR-29a, hsa-miR-29b, hsa-miR-513a-5p and hsa-miR628-3p) was identified. ${ }^{15}$ A single miRNA is able to control the expression of many target genes. Therefore, we investigated the impact of the 4 dysregulated miRNAs so-identified on the global gene expression and identified 64 putative target genes 
of such miRNAs were identified by bioinformatics. ${ }^{15}$ The 64 predicted target genes are involved in important biological processes such as gene expression regulation, proliferation and DNA modification. Very interestingly, among differentially expressed genes, some, such as $M Y C N$ and the DNMT family of proteins, were of particular interest, with $M Y C N$ being a homologue of $M Y C$ and the DNMT proteins being reported to be altered in cancer very frequently. ${ }^{64}$ DNMTs were reported to be specifically upregulated in BL cases lacking the translocation, suggesting that an aberrant epigenetic control occurs in this set of BL cases. As discussed earlier, interaction of Myc with DNMT family members can influence chromatin conformation and subsequent accessibility to RNA polymerase for transcription. ${ }^{22}$ Deregulation of DNMTs by the miR-29 family has also been recently described in another study, which highlights the importance of epigenetic regulation in BL. ${ }^{65} \mathrm{In}$ particular, this study shows hypermethylation of p16 following overexpression of DNMTs, which might favor cell proliferation due to lack of control on cell cycle. ${ }^{65}$ Of great interest is the finding that $M Y C N$ overexpression occurs only in cases lacking $M Y C$ translocation. $M Y C N$ expression is usually not detected in $\mathrm{BL}$ cases, but its deregulation is frequently observed in other cancers, such as neuroblastoma, where there is a different genetic mechanism (amplification of the $M Y C$ gene) which is responsible for over-expression of the Myc protein. ${ }^{66} \mathrm{High}$ expression of $M Y C N$ in cases lacking $M Y C$ translocation may indicate the existence of an alternative cooperative mechanism ensuring high expression of members of the $M Y C$ family in the absence of genetic lesions involving $M Y C$. Very interestingly, deregulation of two of the differentially expressed miRNAs (miR-513a-5p and miR-628-3p) has been recently described in human neuroblastoma, ${ }^{67,68}$ with the miR-628-3p expression correlating with the prognosis of this tumor. ${ }^{69}$

\section{MYC pathway in BL: downregulated miRNAs target genes belonging to the MYC pathway}

Several genes are transcriptionally controlled by Myc, and the existence of an $M Y C$ pathway has been identified in cells. As Myc is highly expressed in BL, it was investigated to what extent the Myc-regulated pathway was affected in BL and through which mechanisms. Results from a previous research study report that $M Y C$ target genes are upregulated in $\mathrm{BL}$ or gamma-irradiated mice tumors. ${ }^{70}$ In this study, 41 miRNAs were found to be downregulated in gamma-irradiated mice lymphomas and 17 miRNAs in BL, resulting in upregulation of miRNA target genes. Interestingly, an enrichment of the $M Y C$ pathway was observed among upregulated genes, thus suggesting that upregulation of $M Y C$ pathway may be a consequence of transcriptional repression of specific miRNAs. ${ }^{70}$

\section{miRNA expression profile: a BL signature for differential diagnosis}

$\mathrm{BL}$ is a very homogeneous entity in terms of gene expression and has a distinctive, unique pattern, which distinguishes it from any other B-cell lymphomas. ${ }^{11,12}$ This observation is extremely useful for diagnostic purposes, as we can classify borderline cases or cases with a histologic "Burkitt-like" presentation based on their distinctive molecular profile. ${ }^{11,12}$ Despite showing some subtle differences in their miRNA profile, only a few differences can be identified at the gene expression level in cases with or without $M Y C$ translocation after enrichment, yet indicating a very high homogeneity of these tumors. The three clinical forms of BL share the same molecular signature, although differences in gene expression can be observed between $\mathrm{EBV}^{+}$and $\mathrm{EBV}^{-} \mathrm{BL}$ cases. ${ }^{71}$ However, such variations may be attributed to the presence of the virus rather than to differences between clinical variants. ${ }^{71}$ The miRNA profile of BL was analyzed and compared to that of diffuse large B-cell lymphoma (DLBCL); a signature of 38 miRNAs was identified, which comprises $M Y C$-regulated and nuclear factor-kB-associated miRNAs. ${ }^{13}$ The same study also reported that only six miRNAs were differentially expressed between eBL and $\mathrm{sBL}$, thus reinforcing the notion that $\mathrm{BL}$ is a very homogeneous entity and its molecular uniqueness can be used for differential diagnosis with other B-cell lymphomas. ${ }^{13}$

More recently, additional studies have confirmed the efficacy of miRNA profile for differential diagnosis and have proved that its reliability can be comparable to gene expression profile results, so far considered the "gold standard" for molecular analyses. A recent study identified a 27-miRNA signature able to distinguish BL from DLBCL, which could also be validated in formalin-fixed paraffin-embedded cases, thus even reinforcing its possible diagnostic application. ${ }^{72}$ Another research identified by deep sequencing the existence of a 22-miRNA signature, which could be used to discriminate BL from DLBCL and follicular lymphomas, again highlighting the importance of molecular profiles for differential diagnosis. ${ }^{73}$

Noteworthy, low or no expression of hsa-miR-155 (or its precursor $B I C$ ) was detected in $\mathrm{BL}$, as reported by several studies, ${ }^{7-76}$ despite this miRNA being one of the most commonly upregulated in B-cell lymphomas. ${ }^{77-79}$ It has been recently demonstrated that low levels of this miRNA determine an increased expression of $A I C D A$, which increases the frequency of $M Y C$ translocation. ${ }^{80}$ Intriguingly, a study shows that higher expression of miR-155 can be found in a 
subset of EBV-positive BL cell lines expressing a viral latency III program, which is usually not detected in primary BLs, whereas low or no expression of this miRNA was detected in EBV-positive BL cases expressing a latency I program, which represent the vast majority of BLs. ${ }^{75}$ It has sometimes been speculated that EBV infection may promote $M Y C$ translocation in BL. This observation may suggest a possible mechanism leading to $M Y C$ translocation in EBV-positive cases by maintaining low miR-155 and consequently inducing $A I C D A$ expression. Interestingly, downregulation of this miRNA can also be used for differential diagnosis, ${ }^{81}$ as high expression of miR-155 is normally reported in other B-cell tumors, where it seems to have a clinical significance as it is associated with chemotherapy failure in DLBCL. ${ }^{72}$

Tables 1 and 2 list the miRNAs whose expression has been reported to be unbalanced in BL.

\section{Virus-encoded miRNAs and deregulation of host cell gene expression: contribution of EBV}

EBV is very often associated with BL, especially in its endemic form. Despite an extensive discussion about this virus, with its latency programs and pathogenetic mechanisms being beyond the aim of this review, it is worth mentioning that EBV may contribute to the pathogenesis of BL through its encoded proteins (genes and viral miRNAs). In BL cells, EBV expresses a latency I program, in which EBNA1 is the only viral protein expressed. Previous studies have demonstrated the role of this protein in the pathogenesis of BL. ${ }^{82,83} \mathrm{EBV}$ also encodes for 44 mature viral miRNAs (viRNAs) belonging to two families (BART and BHRF). ${ }^{84}$ Expression of these viral-encoded miRNAs is also latency regulated, and only a few viRNAs belonging to the BART family are detected in BL. ${ }^{85}$ It has also been demonstrated that three members of the BART family (BART-1-3p, BART5-5p and BART-22-3p) exhibit high similarity with cellular miRNAs, including the miR-29 family (Figure 2B). ${ }^{86-91}$ The expression of viRNAs should be carefully monitored as they compete with cellular miRNAs for the same target genes in the host cell. An interesting study has shown that in a BL-derived cell line expressing a latency III program, EBV-encoded miRNAs target the same genes as the miR17-92 cluster (Figure 2A). ${ }^{92}$ Despite the BL primary tumors mostly expressing a latency I program, this observation is of

Table I List of miRNAs reported to be downregulated in BL

\begin{tabular}{lc}
\hline Downregulated miRNAs & References \\
\hline hsa-miR-22I, hsa-miR-I55, hsa-miR-I46a, hsa-miR-I 146b-5p, hsa-miR-26b, hsa-miR-23a, hsa-miR-30d, hsa-miR-I07, hsa- & I3 \\
miR-I03, hsa-miR-222, hsa-miR-26a, hsa-miR-30a, hsa-miR-I42-5p, hsa-miR-23b, hsa-miR-342-3p, hsa-miR-29b, hsa-miR-34b & 57 \\
hsa-let-7a, hsa-miR-34b & 58 \\
hsa-miR-9* & 15 \\
hsa-miR-29a, hsa-miR-29b & 72 \\
hsa-I55, hsa-I96b, hsa-885-5p, hsa-222, hsa-I35b, hsa-2I, hsa-3I, hsa-708, hsa-23a, hsa-455-5p, hsa-455-3p, hsa-29b, hsa-29c, \\
hsa-342-5p, hsa-I46a, hsa-I50 \\
hsa-5I3, hsa-I8b, hsa-I5b, hsa-454-3p, hsa-I48a, let-7f, hsa-98, hsa-363, hsa-582, hsa-I46, hsa-I55, let-7d, hsa-26b, hsa-29b, \\
hsa-I42-3p, hsa-I6, hsa-I5a, hsa-590, hsa-32, hsa-33I, hsa-I38, hsa-28 \\
hsa-miR-664-3p, hsa-miR-664-5p, hsa-miR-I50-3p, hsa-miR-I50-5p, hsa-miR-I55-5p, hsa-miR-I84, hsa-miR-I96b-5p, hsa-miR- \\
I5Ib, hsa-miR-2III-5p, hsa-miR-22I-3p, hsa-miR-29c-5p & 70 \\
hsa-miR-I55 & 73 \\
\hline
\end{tabular}

Abbreviation: BL, Burkitt lymphoma.

Table 2 List of miRNAs reported to be upregulated in BL

\begin{tabular}{|c|c|}
\hline Upregulated miRNAs & References \\
\hline $\begin{array}{l}\text { hsa-miR-37I-5p, hsa-miR-I85, hsa-miR-93*, hsa-miR-326, hsa-miR-497, hsa-miR-26b*, hsa-miR-339-5p, hsa-miR-485-3p, } \\
\text { hsa-miR-9, hsa-miR-193a-5p, hsa-miR-448, hsa-miR-202*, hsa-miR-483-3p, hsa-miR-26a-I*, hsa-miR-328, hsa-miR-192, hsa- } \\
\text { miR-429, hsa-miR-324-5p, hsa-miR-340, hsa-miR-105*, hsa-miR-I24* }\end{array}$ & 13 \\
\hline hsa-miR-I7-92 & 42 \\
\hline hsa-miR-5I3a-5p, hsa-miR-628-3p & 15 \\
\hline hsa-296-5p, hsa-296-3p, hsa-130b, hsa-18a, hsa-18b, hsa-19a, hsa-19b, hsa-20a, hsa-20b, hsa-17, hsa-106 & 72 \\
\hline hsa-202, hsa-92, hsa-19a, hsa-19b, hsa-296, hsa-663, hsa-22, hsa-320, hsa-18Ib, hsa-422b, hsa-484 & 70 \\
\hline $\begin{array}{l}\text { hsa-miR-17-3p, hsa-miR-18a-3p, hsa-miR-19a-3p, hsa-miR-20a-3p, hsa-miR-25-5p, hsa-miR-93-3p, hsa-miR-106b-3p, hsa-miR- } \\
\text { I06b-5p, hsa-miR-130b-3p, hsa-miR-296-3p, hsa-miR-335-3p, hsa-miR-339-5p, hsa-miR-573, hsa-miR-452। }\end{array}$ & 73 \\
\hline hsa-miR-2I, hsa-miR-23a & 100 \\
\hline
\end{tabular}


interest as it highlights how EBV infection may contribute to deregulate key cellular pathways such as transcription, apoptosis and cell cycle. ${ }^{92}$ Expression of viRNAs could, therefore, result in an aberrant post-transcriptional regulation in infected cells. The presence of EBV can impact on cellular miRNA signature in $\mathrm{BL}^{85,93}$ and on cellular gene expression profile. ${ }^{93}$ An important role for EBV-Bart6 has been suggested, as this miRNA is capable of regulating the expression of PTEN and interleukin-6 receptor complex in infected cells, thus influencing survival and interleukin-6 downstream pathways. ${ }^{85}$ A later study confirmed this finding and highlighted the existence of a synergistic effect between Bart-6 and miR-142, which was previously reported to be upregulated in $\mathrm{BL},{ }^{85}$ to repress $P T E N .{ }^{94}$ This latter observation is of interest as it highlights the active role of EBV in $\mathrm{BL}$ pathogenesis and its interplay with the cellular machinery. It has also been demonstrated that BARTs target Casp3 in $\mathrm{BL}$ and may therefore result in an antiapoptotic effect, thus resulting in a growth advantage for the infected cells. ${ }^{95}$

It is worth mentioning that EBV infection contributes to lymphomagenesis also through mechanisms other than miRNA regulation. We had previously mentioned that a different mutational profile can be observed between eBL and $\mathrm{sBL}$, with endemic cases showing a lower mutation rate. ${ }^{29}$ With the degree of association with EBV being the main difference between the endemic and sporadic variants, it is reasonable to postulate that such difference may be due to the existence of additional pathogenetic mechanisms in EBVpositive cases. ${ }^{29}$ In a recent research paper, the methylome of EBV-positive vs EBV-negative BL-derived cell lines was compared, and the results of this study demonstrated that the presence of the virus is associated with a specific pattern of DNA methylation, suggesting that EBV may contribute to BL pathogenesis through an epigenetic mechanism. ${ }^{96}$ In particular, this paper has demonstrated a higher level of methylation in EBV-positive samples involving, among others, key genes such as ID3 and TCF3 which are usually mutated in sBL, but whose mutation rate is lower in eBL. ${ }^{29}$ Diminished expression of these genes in eBL may, therefore, be a consequence of epigenetic regulation rather than deriving from genetic lesions. This finding suggests that BLs have similar gene expression patterns, but underlying mechanisms may be different and may depend on the presence of EBV. ${ }^{96}$

Although an extensive discussion of other parasites that might act as cofactors in Burkitt lymphomagenesis is beyond the scope of this review, it is worth making a brief comment on another parasite, the protozoon Plasmodium falciparum that causes malaria, as there is a striking overlap in the geographic incidence of eBL and this disease. Even though not much is known about the mechanisms that the parasite uses to contribute to tumor formation, recent literature shows that $P$. falciparum infection drives EBVinfected cells through $\mathrm{GC}$, and it is capable of deregulating the expression of the $A I C D A$ gene (also referred to as $A I D$ ), which would lead to chromosomal translocations, as we mentioned earlier. ${ }^{97-99}$ Translocations would mainly occur in EBV-infected cells within the GC that more likely would tolerate it. This observation reinforces the speculation that other cofactors are required for the occurrence of $\mathrm{BL}$ in endemic areas and that these parasites play an active role in Burkitt lymphomagenesis.

\section{miRNAs as prognostic indicators of $B L$}

Given the clear involvement of miRNA deregulation in the pathogenesis of BL, their diagnostic and prognostic value has been evaluated. A recent study reports that the identification of three circulating miRNAs (miRNA-21, miRNA-23a and miRNA-125b) in the plasma of BL patients may be used as a diagnostic indicator and could be related to clinicopathologic parameters. ${ }^{100}$ Increased expression was observed for miR-21 and miR-23, and it was also correlated with some clinicopathologic parameters (tumor staging, increased white blood cells, increased serum lactate dehydrogenase level, CD10 expression and size of the tumor $>6 \mathrm{~cm}){ }^{100}$ Very interestingly, the expression level of these miRNAs decreased significantly following chemotherapy, suggesting that these miRNAs could be used to monitor therapy efficacy. ${ }^{100}$ Also, an inverse correlation between the level of these miRNAs and patients' outcome was established, indicating these miRNAs act as prognostic indicators as well. ${ }^{100}$ Very recently, the expression of another miRNA (hsa-miR-10a-5p) has been linked to the prognosis of BL patients, with this being downregulated in non-survivors. ${ }^{101}$ Interestingly, genes targeted by this miRNA are involved in control of apoptosis and their overexpression could favor cell growth. Additionally, high expression of CD59 as a result of hsa-miR-10a-5p imbalance may determine reduced sensitivity to chemo- and immunotherapy and explain treatment failure and reduced overall survival in BL. ${ }^{101}$

\section{miRNAs as potential targets in novel treatments for BL}

$\mathrm{BL}$ is classically treated by a combination of chemotherapy and immunotherapy. ${ }^{102}$ Nevertheless, due to the aggressiveness of this tumor, it is imperative to explore more effective therapeutic alternatives. One such possibility would be to 
target miRNAs to either suppress or induce the expression of target genes, which might be relevant for a better prognostic outcome. Recent literature is providing useful information about new drugs or possible new therapeutic targets, including miRNAs. An obvious target for BL treatment would be $M Y C$ and its related network. A recent study reports that the use of INZ(c), a second generation of Inauhzin, is able to suppress Myc expression and it results in inhibition of cell growth in lymphoma cells. ${ }^{103}$ Suppression of $M Y C$ is achieved through the miRNA pathway, as the expression of MYC-targeting miRNAs, such as miR-24 and miR-34a, is induced upon treatment to reduce Myc levels. ${ }^{103}$ Noteworthy, this small molecule does not have considerable side effects and could be used in combination with doxorubicin to reduce Myc expression, allowing the administration of a lower dose of doxorubicin with consequent reduction of side effects. ${ }^{103}$ However, due to the pleiotropic activities regulated by $M Y C$, it is very difficult to design therapeutic approaches to inhibit its expression in human tumors without interfering with its physiological functions, and other potential targets should be explored.

Treatment with the combination of histone deacetylase inhibitor and chemotherapy results in induction of apoptosis in BL cells through the proapoptotic BCL2-related family member Bim protein. ${ }^{104} \mathrm{~A}$ recent study of the same group describes that the use of combination of histone deacetylase inhibitor and chemotherapy could prevent cell growth in $\mathrm{BL}$ by regulating PI3K/Akt, suggesting that other targets, such as the PI3K/Akt signaling network, in addition to $M Y C$ should be further explored. ${ }^{105}$ The combination of demethylating agents and chemotherapy could be used to revert the expression of aberrantly silenced genes and miRNAs, such as p16 and miR-101, miR-143 and miR-145, in BL tumor models. ${ }^{105}$ Of these, miR-145 directly targets MYC and is expressed through the PI3K pathway, which is deregulated in BL. ${ }^{106} \mathrm{Re}$-expression of miR-145 by this combinatorial approach may, therefore, result in reduction of $M Y C$ expression levels. ${ }^{105}$

Regulation of cell proliferation and induction of a more differentiated phenotype could be another possible approach as BL is the fastest growing tumor. It has been recently reported that the re-expression of miR-150 could be used as a possible promising therapeutic target because of its capability of reducing cell proliferation by targeting B-Myb. ${ }^{107,108}$ In addition, this would result in the acquisition of a more differentiated phenotype as BL results from an impairment during differentiation toward plasma cells. ${ }^{109,110}$

\section{Conclusion}

MYC overexpression is the hallmark of BL and it can be consequent to several pathogenetic mechanisms. Recent literature highlights the key role of miRNAs in the pathogenesis of BL that can imbalance Myc and its associated pathways. Detection of miRNA expression can be used for diagnostic and therapeutic purposes. Re-expression of endogenous miRNAs through the administration of demethylating drugs to revert their silencing, or ectopic introduction of exogenous small RNAs that target deregulated genes could represent an exciting alternative to current therapies to improve the overall survival and reduce the side effects in BL patients. However, despite encouraging results, there is still much to uncover before such innovative therapeutic approaches may enter daily practice, and research in the field should be pursued to better clarify their role in the pathogenesis of BL.

\section{Disclosure}

The authors report no conflicts of interest in this work.

\section{References}

1. Burkitt DP. A sarcoma involving the jaws in African children. $\mathrm{Br} J$ Surg. 1958;46:218.

2. Moormann AM, Chelimo K, Sumba OP et al. Exposure to holoendemic malaria results in elevated Epstein-Barr virus loads in children. J Infect Dis. 2005;191:1233-1238.

3. van den Bosch C, Lloyd G. Chikungunya fever as a risk factor for Endemic Burkitt's lymphoma in Malawi. Trans R Soc Trop Med Hyg. 2000;94(6):704-705.

4. Moormann AM, Bailey JA. Malaria - how this parasitic infection aids and abets EBV-associated Burkitt lymphomagenesis. Curr Opin Virol. 2016;20:78-84.

5. Epstein MA, Barr YM. Cultivation in vitro of human lymphoblasts fromBurkitt's malignant lymphoma. Lancet. 1964;1:252-253.

6. Dalla-Favera R, Bregni M, Erikson J, Patterson D, Croce CM. Human c-myc oncgene is located on the region of chromosome 8 that is translocated in Burkitt lymphoma cells. Proc Natl Acad Sci USA. 1982;79:7824-7827.

7. Taub R, Kirsch I, Morton C, Lenoir G, Aaronson S, Leder P. Translocation of the c-myc gene into the immunoglobulin heavy chain locus in human Burkitt lymphoma and murine plasmacytoma cells. Proc Natl Acad Sci USA. 1982;79:7837-7841.

8. Oettgen HF, Clifford P, Burkitt D. Malignant lymphoma involving the jaw in African children: treatment with alkylating agents and actinomycin. Cancer Chemother Rep. 1963;28:25-34.

9. Shannon-Lowe C, Rickinson AB, Bell A. Epstein-Barr virus-associated lymphomas. Philos Trans R Soc Lond B Biol Sci. 2017;372(1732): 20160271

10. Leoncini L, Raphael M, Stein H, Harris NL, Jaffe ES, Kluin PM. Burkitt lymphoma. In: WHO Classification of Tumours of Haematopoietic and Lymphoid Tissues. 4th ed. Edited by International Agency for Research on Cancer. Lyon, France: IARC Press. 2008;262-264. Revised edition published in 2016.

11. Hummel M, Bentink S, Berger H, et al; Molecular Mechanisms in Malignant Lymphomas Network Project of the Deutsche Krebshilfe. A biologic definition of Burkitt's lymphoma from transcriptional and genomic profiling. $N$ Engl J Med. 2006;354:2419-2430. 
12. Dave SS, Fu K, Wright GW, et al; Lymphoma/Leukemia Molecular Profiling Project. Molecular diagnosis of Burkitt's lymphoma. N Engl J Med. 2006;354:2431-2442.

13. Lenze D, Leoncini L, Hummel M, et al. The different epidemiologic subtypes of Burkitt lymphoma share a homogenous micro RNA profile distinct from diffuse large B-cell lymphoma. Leukemia. 2011;25:1869-1876.

14. van Rijk A, Mason D, Jones M, et al. Translocation detection in lymphoma diagnosis by split8 signal FISH: a standardised approach. J Hematop. 2008;1:119-126.

15. De Falco G, Ambrosio MR, Fuligni F, et al. Burkitt lymphoma beyond MYC translocation: N-MYC and DNA methyltransferases dysregulation. BMC Cancer. 2015;15:668-680.

16. Bellan C, Lazzi S, Hummel M, et al. Immunoglobulin gene analysis reveals 2 distinct cells of origin for EBV-positive and EBV-negative Burkitt lymphomas. Blood. 2005;106:1031-1036.

17. Luzzi A, Morettini F, Gazaneo S, et al. HIV-1 Tat induces DNMT over-expression through microRNA dysregulation in HIV-related non Hodgkin lymphomas. Infect Agent Cancer. 2014;9:41-57.

18. Eilers M, Eisenman RN. Myc's broad reach. Genes Dev. 2008;22(20): 2755-2766.

19. Hayward WS, Neel BG, Astrin SM. Activation of a cellular onc gene by promoter insertion in ALV-induced lymphoid leukosis. Nature. 1981;290(5806):475-480.

20. Kalkat M, De Melo J, Hickman KA, et al. $M Y C$ deregulation in primary human cancers. Genes (Basel). 2017;8(6):E151.

21. Pelengaris S, Khan M, Evan G. c-MYC: more than just a matter of life and death. Nat Rev Cancer. 2002;2(10):764-776.

22. Herkert B, Eilers M. Transcriptional repression: the dark side of myc. Genes Cancer. 2010;1(6):580-586.

23. Greenougha A, Dave SS. New clues to the molecular pathogenesis of Burkitt lymphoma revealed through next-generation sequencing. Curr Opin Hematol. 2014;21:326-332.

24. Love C, Sun Z, Jima D, et al. The genetic landscape of mutations in Burkitt lymphoma. Nat Genet. 2012;44:1321-1325.

25. Richter J, Schlesner M, Hoffmann S, et al. Recurrent mutation of the ID3 gene in Burkitt lymphoma identified by integrated genome, exome and transcriptome sequencing. Nat Genet. 2012;44:1316-1320.

26. Schmitz R, Young RM, Ceribelli M, et al. Burkitt lymphoma pathogenesis and therapeutic targets from structural and functional genomics. Nature. 2012;490:116-120.

27. Sander S, Calado DP, Srinivasan L, et al. Synergy between PI3K signaling and MYC in Burkitt lymphomagenesis. Cancer Cell. 2012;22:167-179.

28. Shain AH, Pollack JR. The spectrum of SWI/SNF mutations, ubiquitous in human cancers. PLoS One. 2013;8:e55119.

29. Abate F, Ambrosio MR, Mundo L, et al. Distinct viral and mutational spectrum of Endemic Burkitt lymphoma. PloS Pathog. 2015;11(10): e1005158.

30. Garcia JL, Hernandez JM, Gutierrez NC, et al. Abnormalities on 1q and $7 \mathrm{q}$ are associated with poor outcome in sporadic Burkitt's lymphoma. A cytogenetic and comparative genomic hybridization study. Leukemia. 2003;17:2016-2024.

31. Salaverria I, Zettl A, Beà S, et al; Leukemia and Lymphoma Molecular Profiling Project (LLMPP). Chromosomal alterations detected by comparative genomic hybridization in subgroups of gene expressiondefined Burkitt's lymphoma. Haematologica. 2008;93:1327-1334.

32. Roug AS, Wendtland P, Bendix K, Kjeldsen E. Supernumerary isochromosome 1, $\operatorname{idic}(1)(\mathrm{p} 12)$, leading to tetrasomy 1q in Burkitt lymphoma. Cytogenet Genome Res. 2014;142:7-13.

33. Schiffman JD, Lorimer PD, Rodic V, et al. Genome wide copy number analysis of paediatric Burkitt lymphoma using formalin-fixed tissues reveals a subset with gain of chromosome $13 \mathrm{q}$ and corresponding miRNA over expression. Br J Haematol. 2011;155:477-486.

34. Havelange V, Ameye G, Théate I, et al; GFCH (Groupe Francophone de Cytogénétique Hématologique). Patterns of genomic aberrations suggest that Burkitt lymphomas with complex karyotype are distinct from other aggressive B-cell lymphomas with MYC rearrangement. Genes Chromosomes Cancer. 2013;52:81-92.
35. Salaverria I, Martin-Guerrero I, Wagener R, et al. A recurrent 11q aberration pattern characterizes a subset of $M Y C$-negative high-grade B-cell lymphomas resembling Burkitt lymphoma. Blood. 2014;123:1187-1198.

36. Tuna M, Knuutila S, Mills GB. Uniparental disomy in cancer. Trends Mol Med. 2009;15(3):120-128.

37. Scholtysik R, Kreuz M, Klapper W, et al; Molecular Mechanisms in Malignant Lymphomas Network Project of Deutsche Krebshilfe. Detection of genomic aberrations in molecularly defined Burkitt's lymphoma by array-based, high resolution, single nucleotide polymorphism analysis. Haematologica. 2010;95(12), 2047-2055.

38. Fire A, Xu S, Montgomery MK, Kostas SA, Driver SE, Mello CC. Potent and specific genetic interference by double-stranded RNA in Caenorhabditis elegans. Nature. 1998;391:806-811.

39. Calin GA, Sevignani C, Dumitru CD, et al. Human microRNA genes are frequently located at fragile sites and genomic regions involved in cancers. Proc Natl Acad Sci USA. 2004;101:2999-3004.

40. Chang TC, Yu D, Lee YS, et al. Widespread microRNA repression by Myc contributes to tumorigenesis. Nat Genet. 2008;40(1):43-50.

41. Robertus JL, Kluiver J, Weggemans C, et al. MiRNA profiling in B non-Hodgkin lymphoma: a MYC-related miRNA profile characterizes Burkitt lymphoma. Br J Haematol. 2010;149, 896-918.

42. O’Donnell KA, Wentzel EA, Zeller KI, Dang CV, Mendell JT. c-Myc-regulated microRNAs modulate E2F1 expression. Nature. 2005;435(7043):839-843.

43. Bui TV and Mendell JT. Myc: Maestro of MicroRNAs. Genes Cancer. 2010;1(6):568-575.

44. Xiao C, Srinivasan L, Calado DP, et al. Lymphoproliferative disease and autoimmunity in mice with increased miR-17-92 expression in lymphocytes. Nat Immunol. 2008;9(4):405-414.

45. Jin HY, Oda H, Lai M, et al. MicroRNA-17-92 plays a causative role in lymphomagenesis by coordinating multiple oncogenic pathways. EMBO J. 2013;32(17):2377-2391.

46. Ventura A, Young AG, Winslow MM, et al. Targeted deletion reveals essential and overlapping functions of the miR-17 through 92 family of miRNA clusters. Cell. 2008;132(5):875-886.

47. Robaina MC, Faccion RS, Mazzoccoli L, et al. miR-17-92 cluster components analysis in Burkitt lymphoma: overexpression of miR-17 is associated with poor prognosis. Ann Hematol. 2016;95:881-891.

48. Sampson VB, Rong NH, Han J, et al. MicroRNA let-7a down-regulates MYC and reverts MYC-induced growth in Burkitt lymphoma cells. Cancer Res. 2007;67:(20):9762-9770.

49. Hafsi S, Candido S, Maestro R, et al. Correlation between the overexpression of Yin Yang 1 and the expression levels of miRNAs in Burkitt's lymphoma: a computational study. Oncol Lett. 2016;11: 1021-1025.

50. Castellano G, Torrisi E, Ligresti G, et al. The involvement of the transcription factor Yin Yang 1 in cancer development and progression. Cell Cycle. 2009;8:1367-1372.

51. Bonavida B, Huerta-Yepez S, Baritaki S, et al. Overexpression of Yin Yang 1 in the pathogenesis of human hematopoietic malignancies. Crit Rev Oncog. 2011;16:261-267.

52. Vega MI, Jazirehi AR, Huerta-Yepez S, Bonavida B. Rituximabinduced inhibition of YY1 and Bcl-xL expression in Ramos nonHodgkin's lymphoma cell line via inhibition of NF-kappa B activity: role of YY1 and Bcl-xL in Fas resistance and chemoresistance, respectively. J Immunol. 2005;175:2174-2183.

53. Wang J, Stovall DB, Russell GB, et al. Yin Yang 1 plays an essential role in breast cancer and negatively regulates p27. Am J Pathol. 2012;180: 2120-2133.

54. Wu S, Murai S, Kataoka K, Miyagishi M. Cooperative regulation of p73 promoter by Yin Yang 1 and E2F1. Nucleic Acids Symp Ser (Oxf) 2007;51:347-348.

55. Grönroos E, Terentiev AA, Punga T, Ericsson J. YY1 inhibits the activation of the $\mathrm{p} 53$ tumor suppressor in response to genotoxic stress Proc Natl Acad Sci USA. 2004;101:12165-12170.

56. Libra M, Torrisi E, Castellano G, et al. Computational evaluation of Yin Yang 1 transcript levels in the spectrum of B-cell. Neoplasia. Immunopathol Dis Therap. 2010;1:115-125. 
57. Leucci E, Cocco M, Onnis A, et al. Altered expression of c-mycregulating miRNAs in MYC negative endemic Burkitt lymphoma cases. J Pathol. 2008;216:440-450.

58. Onnis A, De Falco G, Antonicelli G, et al. Alteration of microRNAs regulated by c-Myc in Burkitt lymphoma. PLoS One. 2010;5(9):e12960.

59. Leone G, DeGregori J, Sears R, Jakoi L, Nevins JR. Myc and Ras collaborate in inducing accumulation of active cyclin $\mathrm{E} / \mathrm{Cdk} 2$ and E2F. Nature. 1997;387:422-426.

60. Coller HA, Forman JJ, Legesse-Miller A. "Myc'ed messages": myc induces transcription of E2F1 while inhibiting its translation via a microRNA polycistron. PLoS Genet. 2007;3(8):e146.

61. Hossain A, Kuo MT, Saunders GF. Mir-17-5p regulates breast cancer cell proliferation by inhibiting translation of AIB1 mRNA. Mol Cell Biol. 2006;26(21):8191-9201.

62. Sylvestre Y, De Guire V, Querido E, et al. An E2F/miR-20a autoregulatory feedback loop. J Biol Chem. 2007;282:2135-2143.

63. Bueno MJ, Gomez de Cedron M, Laresgoiti U, Fernandez-Piqueras J, Zubiaga AM, Malumbres M. Multiple E2F-induced microRNAs prevent replicative stress in response to mitogenic signaling. MolCell Biol. 2010;30(12):2983-2995.

64. Zhang W, Xu J. DNA methyltransferases and their roles in tumorigenesis. Biomark Res. 2017;5:1.

65. Robaina MC, Mazzoccoli L, Arruda VO, et al. Deregulation of DNMT1, DNMT3B and miR-29s in Burkitt lymphoma suggests novel contribution for disease pathogenesis. Exp Mol Pathol. 2015;98:200-207.

66. Strieder V, Lutz W. Regulation of N-myc expression in development and disease. Cancer Lett. 2002;180:107-119.

67. Muthu M, Cheriyan VT, Munie S, et al. Mechanisms of neuroblastoma cell growth inhibition by CARP-1 functional mimetics. PLoS One. 2014;9(7):e102567.

68. Schulte JH, Schowe B, Mestdagh P, et al. Accurate prediction of neuroblastoma outcome based on miRNA expression profiles. Int $J$ Cancer. 2010;127(10):2374-2385.

69. Schulte JH, Marschall T, Martin M, et al. Deep sequencing reveals differential expression of microRNAs in favorable versus unfavorable neuroblastoma. Nucleic Acids Res. 2010;38(17):5919-5928.

70. Bueno MJ, Gómez de Cedrón M, Gómez-Lo'pez G, et al. Combinatorial effects of microRNAs to suppress the Myc oncogenic pathway. Blood. 2011;117:6255-6266.

71. Piccaluga PP, De Falco G, Kustagi M, et al. Gene expression analysis uncovers similarity and differences among Burkitt lymphoma subtypes. Blood. 2011;117:3596-3608.

72. Iqbal J, Shen Y, Huang X, et al. Global microRNA expression profiling uncovers molecular markers for classification and prognosis in aggressive B-cell lymphoma. Blood. 2015;125:1137-1145.

73. Hezaveh K, Kloetgen A, Bernhart SH, et al. Alterations of microRNA and microRNA-regulated messenger RNA expression in germinal center B-cell lymphomas determined by integrative sequencing analysis. Haematologica. 2016;101(11):1380-1389.

74. Metzler M, Strissel PL, Strick R, et al. Emergence of translocation $\mathrm{t}(9 ; 11)$-positive leukemia during treatment of childhood acute lymphoblastic leukemia. Genes Chromosomes Cancer. 2004;39:167-169.

75. Kluiver J, Haralambieva E, de Jong D, et al. Lack of BIC and MicroRNA miR-155 expression in primary cases of Burkitt lymphoma. Genes Chromosomes Cancer. 2006;45:147-153.

76. Kluiver J, van den Berg A, de Jong D, et al. Regulation of primicroRNA BIC transcription and processing in Burkitt lymphoma. Oncogene. 2007;26, 3769-3776.

77. van den Berg A, Kroesen BJ, Kooistra K, et al. High expression of B-cell receptor inducible gene BIC in all subtypes of Hodgkin lymphoma. Genes Chromosomes Cancer. 2003;37(1):20-28.

78. Eis PS, Tam W, Sun L, et al. Accumulation of miR-155 and BIC RNA in human B cell lymphomas. Proc Natl Acad Sci USA. 2005;102(10): 3627-3632.

79. Kluiver J, Poppema S, de Jong D, et al. BIC and miR-155 are highly expressed in Hodgkin, primary mediastinal and diffuse large B cell lymphomas. J Pathol. 2005;207(2):243-249.
80. Slezak-Prochazka I, Kluiver J, de Jong D, et al. Inhibition of the miR155 target NIAM phenocopies the growth promoting effect of miR-155 in B-cell lymphoma. Oncotarget. 2015;7(3):2391-2400.

81. Zajdel M, Rymkiewicz G, Chechlinska M, et al. miR expression in $M Y C$-negative DLBCL/BL with partial trisomy 11 is similar to classical Burkitt lymphoma and different from diffuse large B-cell lymphoma. Tumor Biol. 2015;36:5377-5388.

82. Leucci E, Onnis A, Cocco M, et al. B-cell differentiation in EBVpositive Burkitt lymphoma is impaired at posttranscriptional level by miRNA-altered expression. Int. J. Cancer. 2010;126:1316-1326.

83. Onnis A, Navari M, Antonicelli G, et al. Epstein-Barr nuclear antigen 1 induces expression of the cellular microRNA hsa-miR-127 and impairing B-cell differentiation in EBV-infected memory B cells. New insights into the pathogenesis of Burkitt lymphoma. Blood Cancer J. 2012;2:e84.

84. De Falco G, Antonicelli G, Onnis A, Lazzi S, Bellan C, Leoncini L. Role of EBV in microRNA dysregulation in Burkitt lymphoma. Semin Cancer Biol. 2009;19:401-406.

85. Ambrosio MR, Navari M, Di Lisio L, et al. The Epstein-Barr encoded BART-6-3p microRNA affects regulation of cell growth and immuno response in Burkitt lymphoma. Infect Agent Cancer. 2014;9:12.

86. Park SY, Lee JH, Ha M, Nam JW, Kim VN. miR-29 miRNAs activate p53 by targeting p 85 alpha and CDC42. Nat Struct Mol Biol. 2009; 16:23-29.

87. Gottwein E, Cullen BR Viral and cellular microRNAs as determinants of viral pathogenesis and immunity. Cell Host Microbe. 2008; 3:375-387.

88. Lung RW, Tong JH, Sung YM, et al. Modulation of LMP2A expression by a newly identified Epstein-Barr virus-encoded microRNA miRBART22. Neoplasia. 2009;11:1174-1184.

89. Zhu JY, Pfuhl T, Motsch N, et al. Identification of novel Epstein-Barr virus microRNA genes from nasopharyngeal carcinomas. $J$ Virol. 2009;83:3333-3341.

90. Bentwich I, Avniel A, Karov Y, et al. Identification of hundreds of conserved and nonconserved human microRNAs. Nat Genet. 2005;37: 766-770.

91. Landgraf P, Rusu M, Sheridan R, et al. A mammalian microRNA expression atlas based on small RNA library sequencing. Cell. 2007;129: 1401-1414.

92. Riley KJ, Rabinowitz GS, Yario TA, Luna JM, Darnell RB, Steitz JA. EBV and human microRNAs co-target oncogenic and apoptotic viral and human genes during latency. EMBO. 2012;31:2207-2221.

93. Piccaluga PP, Navari M, De Falco G, et al. Virus-encoded microRNA contributes to the molecular profile of EBV-positive Burkitt lymphomas. Oncotarget. 2015;7(1):224-240.

94. Zhou L, Bu Y, Liang Y, Zhang F, Zhang H, Li S. Epstein-Barr virus (EBV)-BamHI-A Rightward Transcript (BART)-6 and cellular microRNA-142 synergistically compromise immune defense of host cells in EBV-positive Burkitt lymphoma. Med Sci Monit. 2016;22: 4114-4120.

95. David T. Vereide, Eri Seto, Ya-Fang Chiu, et al. Epstein-Barr virus maintains lymphomas via its miRNAs. Oncogene. 2014;33(10): $1258-1264$.

96. Hernandez-Vargas H, Gruffat H, Cros MP, et al. Viral driven epigenetic events alter the expression of cancer related genes in EpsteinBarrvirus naturally infected Burkitt lymphoma cell lines. Sci. Rep. 2017; 7:5852-5868.

97. Thorley-Lawson D, Deitsch KW, Duca KA, Torgbor C. The link between Plasmodium falciparum Malaria and Endemic Burkitt's lymphoma-new insight into a 50-year-old Enigma. PLOS Pathog. 2016;12(1):e1005331-e10053315.

98. Torgbor C, Awuah P, Deitsch K, Kalantari P, Duca KA, Thorley-Lawson DA. A multifactorial role for P. falciparum malaria in endemic Burkitt's lymphoma pathogenesis. PLoS Pathog. 2014;10:e1004170.

99. Robbiani DF, Deroubaix S, Feldhahn N, et al. Plasmodium infection promotes genomic instability and AID-Dependent B cell lymphoma. Cell. 2015;162:727-737. 
100. Li J, Zhai X-W, Wang H-S, Qian X-W, Miao H, Zhu X-H. Circulating MicroRNA-21, MicroRNA-23a, and MicroRNA-125b as biomarkers for diagnosis and prognosis of Burkitt lymphoma in children. Med Sci Monit. 2016;22:4992-5002.

101. Oduor CI, Movassag M, Kaymaz Y, et al. Human and Epstein-Barr virus miRNA profiling as predictive biomarkers for Endemic Burkitt lymphoma. Front. Microbiol. 2017;8:501-513.

102. Dozzo M, Carobolante F, Donisi PM, et al. Burkitt lymphoma in adolescents and young adults: management challenges. Adolesc Health Med Ther. 2016;8:11-29.

103. Jung JH, Liao J-M, Zhang Q, et al. Inauhzin(c) Inactivates c-Myc Independently of p53. Cancer Biol Ther. 2015;16(3):412-419.

104. Dos Santos Ferreira AC, Fernandes RA, Kwee JK, Klumb CE. Histone deacetylase inhibitor potentiates chemotherapy-induced apoptosis through Bim upregulation in Burkitt's lymphoma cells. J Cancer Res Clin Oncol. 2012;138(2):317-325.
105. Ferreira AC, Robaina MC, Rezende LM, Severino P, Klumb CE. Histone deacetylase inhibitor prevents cell growth in Burkitt's lymphoma by regulating PI3K/Akt pathways and leads to upregulation of miR143, miR-145, and miR-101. Ann Hematol. 2014;93:983-993.

106. Sachdeva M, Zhu S, Wu F, et al. p53 represses c-Myc through induction of the tumor suppressor miR-145. Proc Natl Acad Sci USA. 2009; 106(9):3207-3212.

107. Chen S, Wang Z, Dai X, et al. Re-expression of microRNA-150 induces EBV-positive Burkitt lymphoma differentiation by modulating c-Myb in vitro. Cancer Sci. 2013;104(7):826-834.

108. Wang M, Yang W, Li M, Li Y. Low expression of miR-150 in pediatric intestinal Burkitt lymphoma. Exp Mol Pathol. 2014;96:261-266.

109. Staudt LM, Dave S. The biology of human lymphoid malignancies revealed by gene expression profiling. Adv Immunol. 2005;87:163-208.

110. Lin KI, Tunyaplin C, Calame K. Transcriptional regulatory cascades controlling plasma cell differentiation. Immunol Rev. 2003;194:19-28.
Blood and Lymphatic Cancer: Targets and Therapy

\section{Publish your work in this journal}

Blood and Lymphatic Cancer: Targets and Therapy is an international peer-reviewed, open access journal focusing on blood and lymphatic cancer research, identification of therapeutic targets and the optimal use of preventative and integrated treatment interventions to achieve improved outcomes, enhanced survival and quality of life for the

\section{Dovepress}

cancer patient. The manuscript management system is completely online and includes a very quick and fair peer-review system. Visit http://www.dovepress.com/testimonials.php to read real quotes from published authors.

Submit your manuscript here: https://www.dovepress.com/blood-and-lymphatic-cancer-targets-and-therapy-journal 